\title{
In vitro and in vivo performance of methacrylated gellan gum hydrogel formulations for cartilage repair*
}

\author{
Carlos A. Vilela (D), 1,2,3,4 Cristina Correia, ${ }^{5}$ Alain da Silva Morais, ${ }^{2,3}$ Tírcia C. Santos (D), ${ }^{2,3}$ \\ Ana C. Gertrudes, ${ }^{5}$ Elsa S. Moreira, ${ }^{5}$ Ana M. Frias, ${ }^{5}$ David A. Learmonth, ${ }^{5}$ Pedro Oliveira, ${ }^{6}$ \\ Joaquim M. Oliveira, ${ }^{2,3}$ Rui A. Sousa, ${ }^{5}$ João D. Espregueira-Mendes, ${ }^{1,2,3,7}$ Rui L. Reis ${ }^{2,3}$ \\ ${ }^{1}$ Life and Health Sciences Research Institute (ICVS), School of Medicine, University of Minho, Braga, Portugal \\ ${ }^{2} 3$ Bs Research Group - Biomaterials, Biodegradables and Biomimetics, University of Minho, Headquarters of the European \\ Institute of Excellence on Tissue Engineering and Regenerative Medicine, Guimarães, Portugal \\ ${ }^{3}$ ICVS/3Bs-PT Government Associate Laboratory, Braga/Guimarães, Portugal \\ ${ }^{4}$ Orthopaedic Department, Hospital da Senhora da Oliveira Guimarães EPE, Guimarães, Portugal \\ ${ }^{5}$ Stemmatters, Biotecnologia e Medicina Regenerativa SA, Guimarães, Portugal \\ ${ }^{6}$ ISUP-EPI Unit, Institute of Biomedical Sciences Abel Salazar (ICBAS), University of Porto, Porto, Portugal \\ ${ }^{7}$ Clínica do Dragão, Espregueira-Mendes Sports Centre, FIFA Medical Centre of Excellence and D. Henrique Research Centre, \\ Porto, Portugal
}

Received 11 December 2017; revised 26 February 2018; accepted 15 March 2018

Published online 10 April 2018 in Wiley Online Library (wileyonlinelibrary.com). DOI: 10.1002/jbm.a.36406

\begin{abstract}
Methacrylated gellan gum (GGMA) formulation is proposed as a second-generation hydrogel for controlled delivery of cartilage-forming cells into focal chondral lesions, allowing immediate in situ retention of cells and 3D filling of lesion volume, such approach deemed compatible with an arthroscopic procedure. Formulation optimization was carried out in vitro using chondrocytes and adipose mesenchymal stromal/stem cells (ASCs). A proof-of-concept in vivo study was conducted using a rabbit model with induced chondral lesions. Outcomes were compared with microfracture or non-treated control. Three grading scores were used to evaluate tissue repair after 8 weeks by macroscopic, histological and immunohistochemical analysis. Intense collagen type II and low collagen type I gene and protein expression were achieved in vitro by the
\end{abstract}

ASC + GGMA formulation, in light with development of healthy chondral tissue. In vivo, this formulation promoted significantly superior de novo cartilage formation compared with the non-treated group. Maintenance of chondral height and integration with native tissue was further accomplished. The physicochemical properties of the proposed GGMA hydrogel exhibited highly favorable characteristics and biological performance both in vitro and in vivo, positioning itself as an attractive xeno-free biomaterial to be used with chondrogenic cells for a cost-effective treatment of focal chondral lesions. (c) 2018 Wiley Periodicals, Inc. J Biomed Mater Res Part A: 106A: 1987-1996, 2018

Key Words: cartilage repair, methacrylated gellan gum, hydrogel, adipose stromal/stem cells, chondrocytes

How to cite this article: Vilela CA, Correia $C$, da Silva Morais A, Santos TC, Gertrudes AC, Moreira ES, Frias AM, Learmonth DA, Oliveira P, Oliveira JM, Sousa RA, Espregueira-Mendes JD, Reis RL. 2018. In vitro and in vivo performance of methacrylated gellan gum hydrogel formulations for cartilage repair. J Biomed Mater Res Part A 2018:106A:1987-1996.

\section{INTRODUCTION}

Complementary efforts for improving cartilage repair $^{1}$ have addressed optimization of combination strategies involving predominantly autologous articular chondrocytes ${ }^{2,3}$ for which decades of performance history have been collected. ${ }^{4-7}$ Robust long-term outcomes is among its major advantages, ${ }^{8,9}$ yet suboptimal cell retention within cartilage lesion sites has been a concern, ${ }^{10-12}$ which has driven the development and marketing of novel scaffolds or matrices to enhance efficacy of these procedures. ${ }^{12-14}$ Nonetheless, most surgical protocols intervene directly on the subchondral bone either for recruitment of cells or for fixation of the scaffold, involve additional fixation systems, or require invasive procedures, such as arthrotomy, to be effectively implanted. ${ }^{15,16}$

In previous works ${ }^{17-19}$ gellan gum (GG) has been proposed as new biomaterial for cartilage tissue engineering applications. Its versatility and efficacy has been demonstrated for cartilage repair strategies involving both subchondral stimulation and cell transplantation using a rabbit model. ${ }^{18}$ Both histological and gene expression outcomes confirmed the potential of this approach for cartilage repair

\footnotetext{
*The author, or one or more of the authors, has received or will receive remuneration or other prequisites for personal or professional use from a commercial or industrial agent in direct or indirect relationship to their authorship.

Correspondence to: C. A. Vilela; e-mail: cvilelagomes@gmail.com

Contract grant sponsor: Portuguese National Innovation Agency; contract grant number: QREN-13/SI/2011-23189
} 
but limitations concerning usability and crosslinking kinetics have been identified, which could limit its translation into a clinical setting. Subsequent work explored alternative synthetic routes to enhance performance of GG hydrogels, namely the methacrylation of the molecule (GGMA) ${ }^{20-22}$ for other biomedical applications. Rational design modification of GG yielded a second-generation GGMA polymer endowed with improved physicochemical characteristics, including better solubilization, liquid formulation prior to injection at room temperature, improved gelification kinetics, and more robust mechanical properties of the hydrogel ${ }^{20-22}$ the latter being greatly dependant on the crosslinking mechanism. In an applied perspective, the adoption of an injectable formulation based in GGMA in the context of cartilage repair is highly attractive, as its solution-state properties make it potentially compatible with minimally invasive procedures.

Given the positive track-record of the parent GG molecule on what regards safety and performance, it becomes mandatory to quantify the actual benefits of GGMA in the cartilage repair application context. Understanding physicochemical performance of GGMA could be explored to simplify the surgical protocol, to improve delivery and functional commitment of cells employed, as well as to minimize damage of the subchondral compartment during the surgical protocol. In this regard, this study aims to comparatively assess the safety and performance of GG and GGMA by in vitro methods, as well as to characterize the performance of GGMA hydrogel as vehicle for delivery and retention of chondrogenic cells within chondral lesions, by assessing functional development of hyaline cartilage tissue in a rabbit model.

On the perspective of functional performance of cells, the risk of chondrocyte de-differentiation, ${ }^{23}$ or lack of potency of the autologous chondrocytes ${ }^{24-26}$ along the need for double surgery and prolonged surgical pre-planning, has inspired the study of alternative cell sources, including mesenchymal stromal/stem cells (MSCs) in general, and adipose-derived stromal/stem cells (ASCs) in particular. ${ }^{17,18,27}$ As compared with other MSC sources, adipose tissue can be harvested with reduced morbidity at the donor site and yields of ASC are considerably high. ${ }^{28}$ The immunomodulatory and anti-inflammatory properties of ASC makes them an especially attractive cell source for development of off the shelf regenerative medicine treatments. ${ }^{27,29,30}$

Herein, preliminary screening demonstrated improved cell viability of ASC within ionic-crosslinked GGMA as compared with photo-crosslinked, therefore favoring further experimentation with ionically crosslinked GGMA. The best performing combination was further evaluated for the treatment of focal chondral lesions in a rabbit model, by adopting a physiologically inspired crosslinking approach devoid of toxic photo-initiators and electromagnetic radiation sources, which is highly desirable from both regulatory and surgical protocol perspectives.

\section{MATERIALS AND METHODS}

In vitro chondrogenesis

Preparation of purified GG and GGMA. Commercial GG (GGc) (Gelzan, Sigma-Aldrich) was purified according to the method described by Doner ${ }^{31}$ with several modifications. Briefly, GGc was suspended in distilled water $(1 \% \mathrm{w} / \mathrm{V})$ and warmed to $60^{\circ} \mathrm{C}$ with stirring. To this solution was added Amberlite IR-120 $\left(\mathrm{H}^{+}\right.$form) (Sigma-Aldrich) until $\mathrm{pH} 2.5$. The suspension was filtered and aqueous sodium hydroxide $(\mathrm{NaOH}, 1 \mathrm{~N})$ was added until $\mathrm{pH} 8$, while stirring. The resulting solution was filtered and the filtrate poured onto absolute ethanol (1 L), forming a thick fibrous precipitate. After $1 \mathrm{~h}$, the precipitate was filtered, washed with absolute ethanol and dissolved in distilled water. The resulting solution was transferred to a cellulose membrane (Molecular weight cut-off (MWCO) $12 \mathrm{kDa}$ ) and dialyzed against distilled water for 3 days. After freezing $\left(-20^{\circ} \mathrm{C}\right)$ and lyophilization, the purified GG (GGp) was obtained. GGMA, with a degree of substitution with methacrylate groups between 1.5 and 5\% was prepared as follows: GGc was dissolved in water to give a solution of $1 \% \mathrm{w} / \mathrm{V}$ concentration. Heating was stopped and the solution $\mathrm{pH}$ was adjusted to 8.5 by $\mathrm{NaOH}(1 N)$. Thereupon, excess glycidyl methacrylate was added in one portion and the methacrylation reaction was allowed to proceed for $24 \mathrm{~h}$ whilst maintaining the solution $\mathrm{pH}$ close to 8.5. Acetone was then added to the reaction mixture which was allowed to stand for $2 \mathrm{~h}$. The precipitate was recovered by filtration, dissolved in distilled water and then placed in a cellulose dialysis membrane (MWCO 12 $\mathrm{kDa}$ ) and dialyzed against distilled water for 7 days. The dialyzed solution was then frozen at $-20^{\circ} \mathrm{C}$ and subsequently freeze-dried to give GGMA as an amorphous white solid. For hydrogel preparation, GGp and GGMA powder were dissolved in deionized water to achieve solutions at 1.25 and $2.5 \% \mathrm{w} / \mathrm{V}$, respectively. Dissolution was effected at $37^{\circ} \mathrm{C}$ in a water-bath with $100 \mathrm{rpm}$ agitation.

In vitro culture of human cells. Human nasal cartilage (hNC) was obtained with informed consent, as surgical waste from a local hospital and further processed for isolation of chondrocytes as described elsewhere. ${ }^{17}$ Chondrocytes where thawed and expanded in DMEM:F12 supplemented with $10 \% \mathrm{v} / \mathrm{v}$ FBS and $1 \% \mathrm{v} / \mathrm{v}$ antibioticantimycotic (Gibco, USA) until passage 3. Human adipose tissue (hAT) was obtained from liposuction procedures, after informed consent and medical questionnaire according to European directives. Collection of adipose samples was approved by the Institutional Review Board of "Centro Hospitalar de São João", Portugal. Briefly, hAT was washed with a decontamination solution (Base-128 Alchimia, Italy) and digested with collagenase $(0.4 \mathrm{U} / \mathrm{mL}$, NB6, SERVA, Germany) for $1 \mathrm{~h}$ at $37^{\circ} \mathrm{C}$ with agitation. The stromal vascular fraction (SVF) was collected after purification steps that include washing, centrifugation and lysis of red blood cells. Human adipose mesenchymal stromal/stem cells (hASC) were obtained from SVF by plating and further expansion in low serum media (MesenPro, Gibco, USA) or xeno-free media (Fibrolife, Lifeline, USA) until passage 2 or 4 . Quality control included validation of MSC immunophenotype (CD31, CD34, CD45, CD73, CD90, and CD105, BD Biosciences, USA) characterized by flow cytometry analysis (FACS Canto, FACSDiva software, antibodies BD Biosciences, USA), and trilineage 


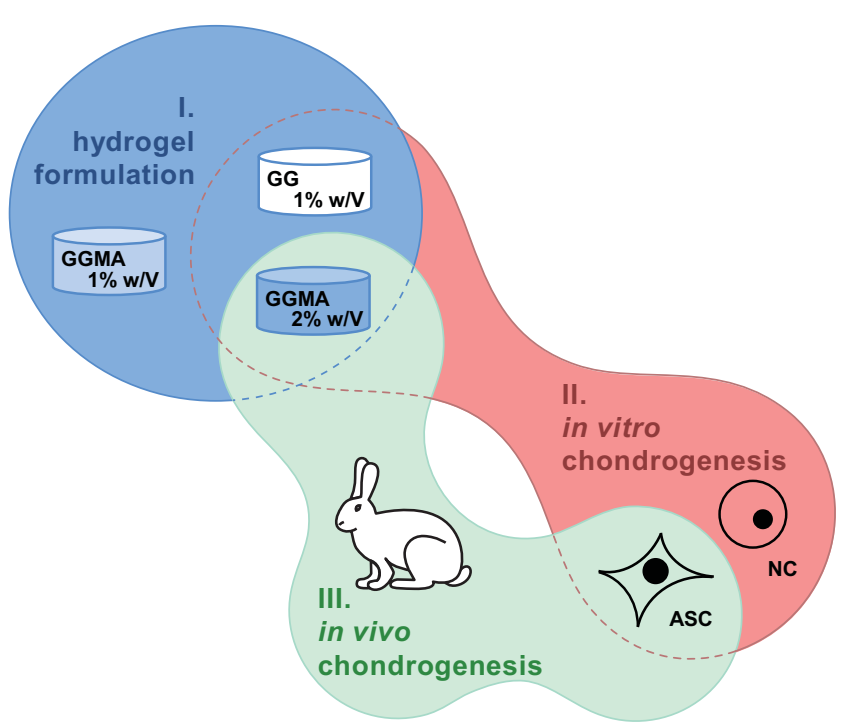

FIGURE 1. Schematic representation of experimental design. Hydrogel formulations based on GG and its methacrylated derivative (GGMA) were tested for gelification and cell encapsulation (stage I). Two formulations were selected for in vitro assessment of chondrogenesis (stage II) using nasal chondrocytes (NC) and ASC. A final formulation was applied for treatment of focal chondral lesions in an induced rabbit model (stage III).

differentiation (StemPro, Gibco, USA) identified by alizarin red, oil red $\mathrm{O}$ and alcian blue stainings for osteogenesis, adipogenesis and chondrogenesis, respectively.

Preparation of cell-encapsulated hydrogels for in vitro culture. An initial comparison study was performed to evaluate comparative performance of GG and GGMA with respect to hydrogel formation and metabolic activity of encapsulated hASC. Selected formulations were further used for assessment of chondrogenesis by hASC or hNC (Fig. 1). Cell suspensions were prepared in cell culture media and mixed with GG or GGMA solution in a 2:8 ratio in order to yield a final cell density of $5 \times 10^{6}$ cells $/ \mathrm{mL}$ and hydrogel concentrations of 1 and $2 \%$ $\mathrm{w} / \mathrm{V}$, respectively. Cellular hydrogels of $20 \mu \mathrm{L}$ volume were pipetted (with aid of a positive displacement pipette) into wells of non-adherent cell culture well-plates and covered with culture media for crosslinking. To induce chondrogenesis of hASC, serum-free chondrogenic media (StemPro, Gibco, USA) was used. At specific time-points (i.e., 0, 7, and 21 days), individual hydrogels were collected for analysis. The hASC-GGMA combination was further scaled to hydrogels of $50 \mu \mathrm{L}$ volume containing $10 \times 10^{6}$ cells $/ \mathrm{mL}$ and tested for chondrogenesis.

Assessment of in vitro cell viability and chondrogenesis. Cell metabolic activity was determined at each time-point by MTS assay (Promega USA) and cell viability was further microscopically assessed by Live/Dead assay (calcein AM and propidium iodide [Invitrogen, USA $1 \mathrm{mg} /$ $\mathrm{mL}]$ ). For histology and immunohistochemistry (IHC), hydrogels were fixed (10\% formalin), followed paraffin processing. Histochemical staining of glycosaminoglycans (GAGs) by Safranin 0/Fast green and Alcian Blue were performed as previously described in. ${ }^{17}$ For IHC, reagents from Vector Laboratories (UK) were used. Sections were incubated into recommended antigen retrieval solutions, followed by inhibition of endogenous peroxidases with $0.3 \% \quad \mathrm{H}_{2} \mathrm{O}_{2}$ and blocking with normal horse serum. Thereafter, sections were stained with primary antibody Mouse anti-human Anti-Collagen I or Mouse anti-human Anti-Collagen II (Abcam, UK) for $1 \mathrm{~h}$, RT and a diluted biotinylated secondary antibody solution (VECTASTAIN Elite ABC kit) for $30 \mathrm{~min}$, RT. Signal development was performed with the DAB substrate kit. For gene expression analysis, hydrogels were collected into TRI Reagent (Sigma-Aldrich, USA) and recommended protocol for RNA extraction from tissues was followed. Complementary cDNA was obtained by using the High Capacity cDNA Reverse Transcription Kit. Gene amplification was conducted using TaqMan Fast Advanced Master Mix and TaqMan Gene Expression Assays for Collagen type II (Hs00264051_m1) and Collagen type I (Hs00164004_m1). Glyceraldehyde 3-phosphate dehydrogenase (GAPDH, Hs99999905_m1) was chosen as an invariant standard (housekeeping gene). Quantitative reverse transcription (RTqPCR) analysis was carried out with the StepOnePlus RealTime PCR System and software (all reagents and equipment from Applied Biosystems, USA). Results were normalized to GAPDH and expressed as relative gene expression using the $\Delta \Delta \mathrm{Ct}$ method. The expression data were presented as average values for each group $(n=3 \pm \operatorname{SD})$.

\section{In vivo chondrogenesis}

Chondral lesion induction and repair in a rabbit model. The ICRS and ASTM guidelines were followed for a proof-of-concept (PoC) study in rabbits to assess in vivo cartilage tissue repair. $^{32,33}$ All animal procedures were based upon the "3R's" policy, approved by the Institutional Ethical Committee, according to the National authority Guide for the Care and Use of Laboratory Animals. Skeletally mature (12- to 14-weeks old) New Zealand white rabbits $(2.5 \pm 0.25 \mathrm{~kg}$; Charles-River, France, $n=6)$ were used for harvesting adipose tissue and subsequent autologous treatment of focal chondral lesions. Interscapular adipose tissue $(\sim 10 \mathrm{~g})$ was collected under anesthesia with a mixture of ketamine hydrochloride (Imalgene, $25 \mathrm{mg} / \mathrm{kg}$ i.m.) and medetomidine hydrochloride (Domitor, $0.3 \mathrm{mg} / \mathrm{kg}$ i.m.). The obtained adipose tissue samples were digested for $1 \mathrm{~h}$ at $37^{\circ} \mathrm{C}, 100 \mathrm{rpm}$ with Collagenase NB4 Standard Grade 0.2 U/mL (Serva, Germany). After complete digestion, cells were cultured in complete media based on alpha MEM supplemented with $10 \% \mathrm{v} / \mathrm{v}$ FBS and $1 \% \mathrm{v} / \mathrm{v}$ antibiotic-antimycotic (Gibco, USA), until passage 2. One week after adipose tissue harvest, surgery was conducted to create critical chondral defects in the knee for immediate administration of treatment. Rabbits were anesthetized as described above and both knees were shaved and disinfected. An internal para-patelar incision was made to expose the knee. The patella was dislocated and two 4$\mathrm{mm}$ diameter lesions were made in the trochlear grove of each knee using a biopsy punch. Lesion sites were carefully cleaned with a curette to not affect the sub-chondral 

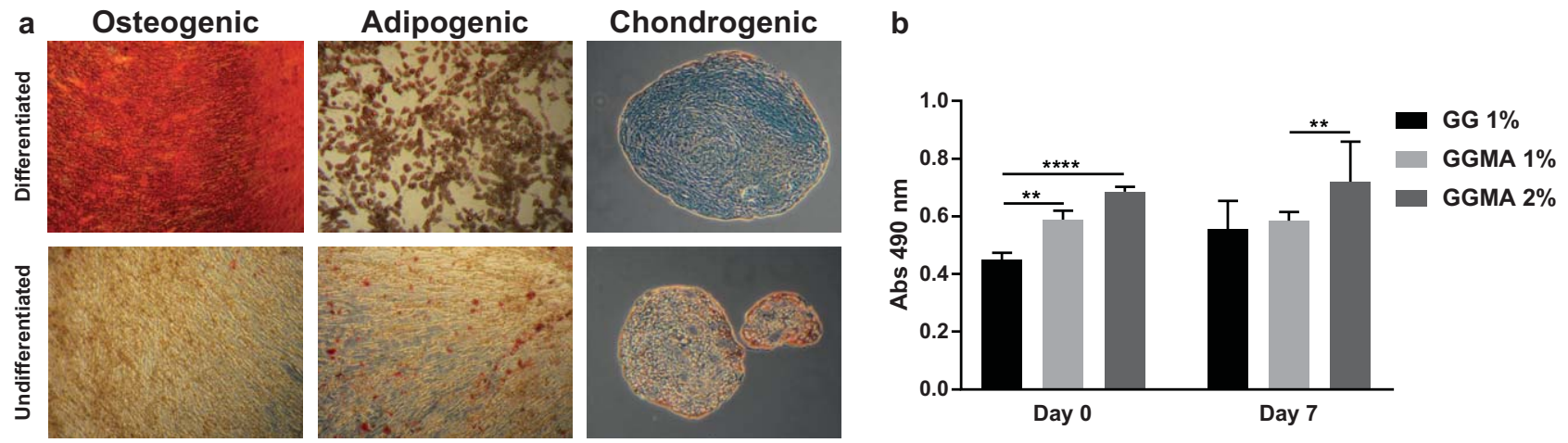

FIGURE 2. Preliminary in vitro studies with hASC. (a) Trilineage differentiation of hASC identified by alizarin red, oil red O and alcian blue stainings for osteogenesis, adipogenesis, and chondrogenesis, respectively. (b) Metabolic activity of hASC encapsulated in GG and GGMA hydrogel formulations ( 1 and $2 \% \mathrm{w} / \mathrm{V}$ ) upon encapsulation (day 0 ) and after 1 week in vitro culture. ${ }^{* *} p<0.01,{ }^{* * * *} p<0.0001$.

bone. Defects were randomly allocated to one of the following experimental conditions: (1) rabbit autologous ASC encapsulated in GGMA hydrogel (GGMA + rASC); (2) microfracture (MFX) (positive control) and iii) empty lesion (negative control). Autologous rabbit ASC were encapsulated in GGMA hydrogels as described earlier $\left(10 \times 10^{6}\right.$ cells $/ \mathrm{mL}, 2 \% \mathrm{w} / \mathrm{V}$ ) immediately before delivery into the chondral defect. In situ crosslinking was promoted with PBS and a setting time of $10 \mathrm{~min}$ was allowed before closure. MFXs were made with a 0.8-mm Kirshner wire (six holes per defect) with 1- to 2-mm depth from which bleeding was observed. Finally, the patella was reduced and the wound was closed. After recovery from surgery, animals were placed in individual cages and fed ad libitum.

Assessment of in vivo cartilage tissue repair. Cartilage regeneration was allowed for 8 weeks, after which animals were anesthetized as described earlier and euthanized (Eutasil, $200 \mathrm{mg} / \mathrm{kg}$ ). In each knee, an internal para-patellar incision was made and the patella carefully dislocated. Macroscopic pictures were taken and explant tissue was harvested with a 6-mm diameter punch in order to collect native tissue surrounding the lesion site, as well as subchondral bone. Explants were paraffin-processed after fixation (10\% formalin) and decalcification (Biodec R, BioOptica, Italy). For IHC, sections were processed as described above, followed by incubation with primary antibody mouse anti-rabbit anti-collagen I (Abcam, UK) or mouse anti-rabbit anti-collagen II (Merck Millipore, USA) for $1 \mathrm{~h}$, RT. Histochemical staining of GAGs was performed by Safranin 0/fast green and three scoring systems were used to assess the quality of cartilage repair, namely O'Driscoll, Pineda and Wakitani. $^{34}$

\section{Statistical analysis}

Results are summarized by mean or median and corresponding standard deviation or interquartile range. For in vitro studies, Student's $t$ test and two-way analysis of variance (ANOVA) were used to evaluate differences among groups. Normality was evaluated by the Shapiro-Wilk test.
When normality or homogeneity of variances was not verified, non-parametric tests were used. For in vivo studies, the histological scores for each specimen were evaluated independently by three observers at three different times. For evaluation, the observers were blinded for the type of treatment and the specimens were randomly allocated to each observer. The comparisons between treatment groups were performed by two-way ANOVA. Since there were no statistical differences between the observers' evaluations, the results were analyzed using one-way ANOVA. In cases where homogeneity of variances was not observed, the Kruskal-Wallis nonparametric test was adopted. Multiple comparisons were based on the Tukey HSD test or the Mann-Whitney test, with the corresponding significance level and Bonferroni correction. Statistical analysis was performed using the GraphPad Prism 4.0c software or IBM SPSS Statistics, version 23. Statistical significance was defined for $p<0.05$.

\section{RESULTS}

\section{In vitro chondrogenesis}

Trilineage differentiation capacity was confirmed for CD73+/CD90+/CD105+/CD31-/CD34-/CD45- hASC, with expressive mineralization, lipid formation, and GAG deposition upon osteogenesis, adipogenesis and chondrogenesis, respectively [Fig. 2(a)]. Upon encapsulation within GGbased hydrogel formulations, higher metabolic activity was observed for hASC encapsulated in GGMA 1 and $2 \% \mathrm{w} / \mathrm{V}$ as compared with GG at $1 \% \mathrm{w} / \mathrm{V}(p<0.01$ and 0.001 , respectively) [Fig. 2(b)]. GG at $2 \% \mathrm{w} / \mathrm{V}$ provided inadequate sol-gel transition time for cell encapsulation studies. After 7 days of culture, highest metabolic activity was observed by cells encapsulated in GGMA $2 \% \mathrm{w} / \mathrm{V}$ $(p<0.01)$ therefore this formulation was selected for further in vitro cell encapsulation studies concurrently with GG 1\% w/V (Fig. 3).

After 21 days of in vitro culture, the viability of chondrocytes and hASC, assessed microscopically by live/dead assay, was comparable between GGp and GGMA hydrogels [Fig. 3(a), top and middle rows]. On the other hand, hASC chondrogenically differentiated within both hydrogel groups 
a
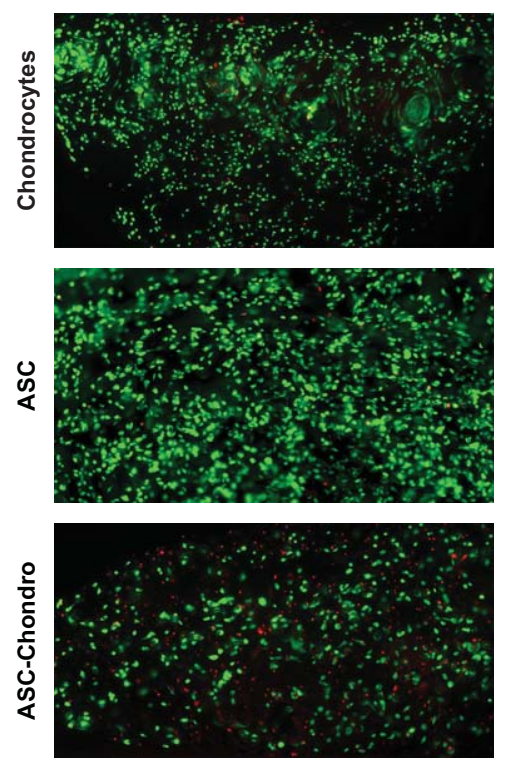

GGMA
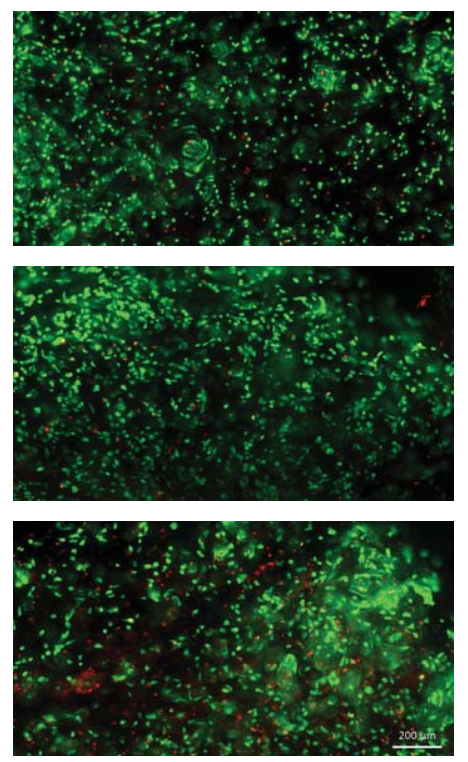

b
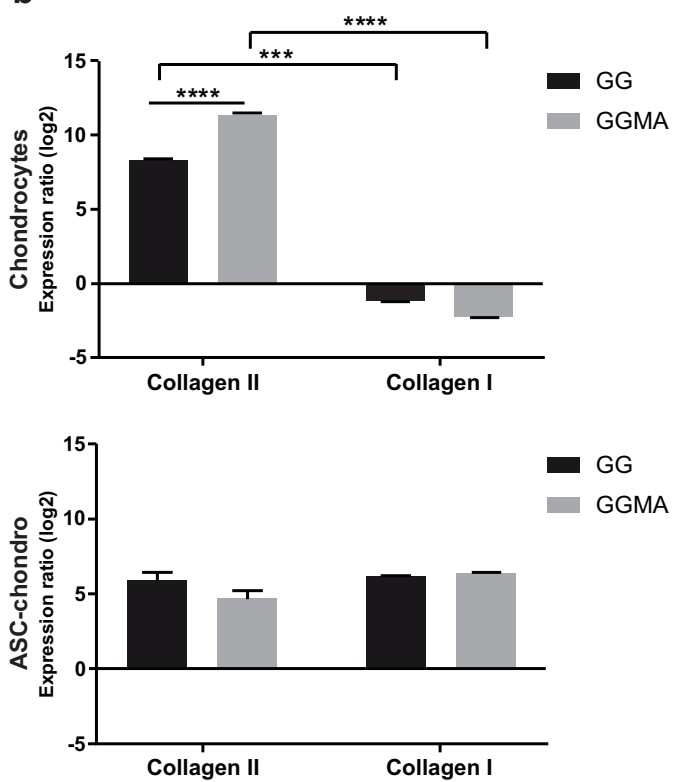

FIGURE 3. In vitro chondrogenesis. (a) Microscopic imaging of encapsulated cells stained by calcein AM (live, green) and propidium iodide (dead, red) upon 21 days in vitro culture in 1\% w/V GG and 2\% w/V GGMA hydrogels. (b) Normalized gene expression ratio (days 21-0) of GG/ GGMA encapsulated chondrocytes (top) and chondrogenically induced hASC (bottom). ${ }^{* * *} p<0.001,{ }^{* * * *} p<0.0001$.

(hASC-chondro) demonstrated increased viability within the GGMA hydrogel [Fig. 3(a), bottom row]. On what regards expression of chondrogenic markers, both formulations favored maintenance of healthy chondrocytes as evidenced by significantly increased expression of collagen type II relative to collagen type I. Such response was superior by the GGMA formulation $(p<0.0001)$ as compared with the $\mathrm{GG}$ $(p<0.001)$. Furthermore, chondrocytes cultured within the GGMA hydrogel presented higher collagen type II expression ratio as compared with the parent GGp hydrogel [Fig. 3(b), top] $(p<0.0001)$. On what regards hASC [Fig. 3(b), bottom], such cells effectively expressed collagen type II upon chondrogenic stimuli (21 days) when cultured within either hydrogel formulation $(p>0.05)$. Concurrently, collagen type I expression was not superior to collagen type II at this time-point $(p>0.05)$. When doubling hASC concentration within the GGMA hydrogel up to $10 \times 10^{6}$ cells $/ \mathrm{mL}$, increasing expression of collagen type II was obtained in the course of chondrogenic differentiation [Fig. 4(a)]. Simultaneously, very low expression of collagen type I was obtained along culture $(p<0.001$ and 0.05 at days 14 and 21 , respectively). Samples were further collected for histological analysis and subsequent identification of extracellular matrix (ECM) components [Fig. 4(b)]. Progressive deposition of healthy chondrogenic ECM was observed as evidenced by safranin $\mathrm{O} /$ fast green staining of cartilage matrix, alcian blue detection of sulfated GAG and IHC of human collagen type II. The absence of collagen type I deposition also indicates development of non-fibrous cartilaginous tissue. Macroscopically, transparent hydrogels at the beginning of culture showed reduction in transparency (not quantified) into an off-white opaque appearance after 3 weeks in vitro culture [Fig. 4(b), bottom row].

\section{In vivo cartilage repair}

At surgical treatment day, expanded rASC were mixed with GGMA solution at time of surgery so as to form a homogenous suspension. Upon injection into the lesion, the viscosity of the suspension allowed spatial control of delivery within the lesion volume, without spill over at the edges of the defect. Gelification was allowed to occur during $10 \mathrm{~min}$ as to assure maintenance of the hydrogel in the lesion site, allowing immediate retention of cells in situ. Rabbits remained healthy during all experimentation period, presenting normal weight gain and absence of signs of infection or disease. From macroscopic observation at time of explant surgery, no apparent abnormalities of the patella position were observed; neither signs of inflammation, abnormalities of the synovium, loose bodies, osteophytes or degenerative process were found. Macroscopic observation showed native cartilage near the defect site as well as the opposing cartilage to be bright and white without visible degenerative signs. In all defects, tissue formation was observed (Fig. 5), and the margin between the defect and the surrounding cartilage were visible, which was more evident for the empty control group. The defects treated with the GGMA + rASC combination showed compact bright tissue filling, despite macroscopic variability observed between defects. The lesions treated by MFX presented an irregular filling of the lesion site, with tissue of a dim appearance. A similar outcome was observed for untreated lesions (empty defects). Tissue explants were further harvested for histological analysis. The quality of cartilage repair was assessed by three scoring systems (Fig. 5), which have inverse scales for indication of cartilage quality and outcome: according to O'Driscoll, high point values indicate enhanced cartilage while according to Pineda and Wakitani, low total point values 


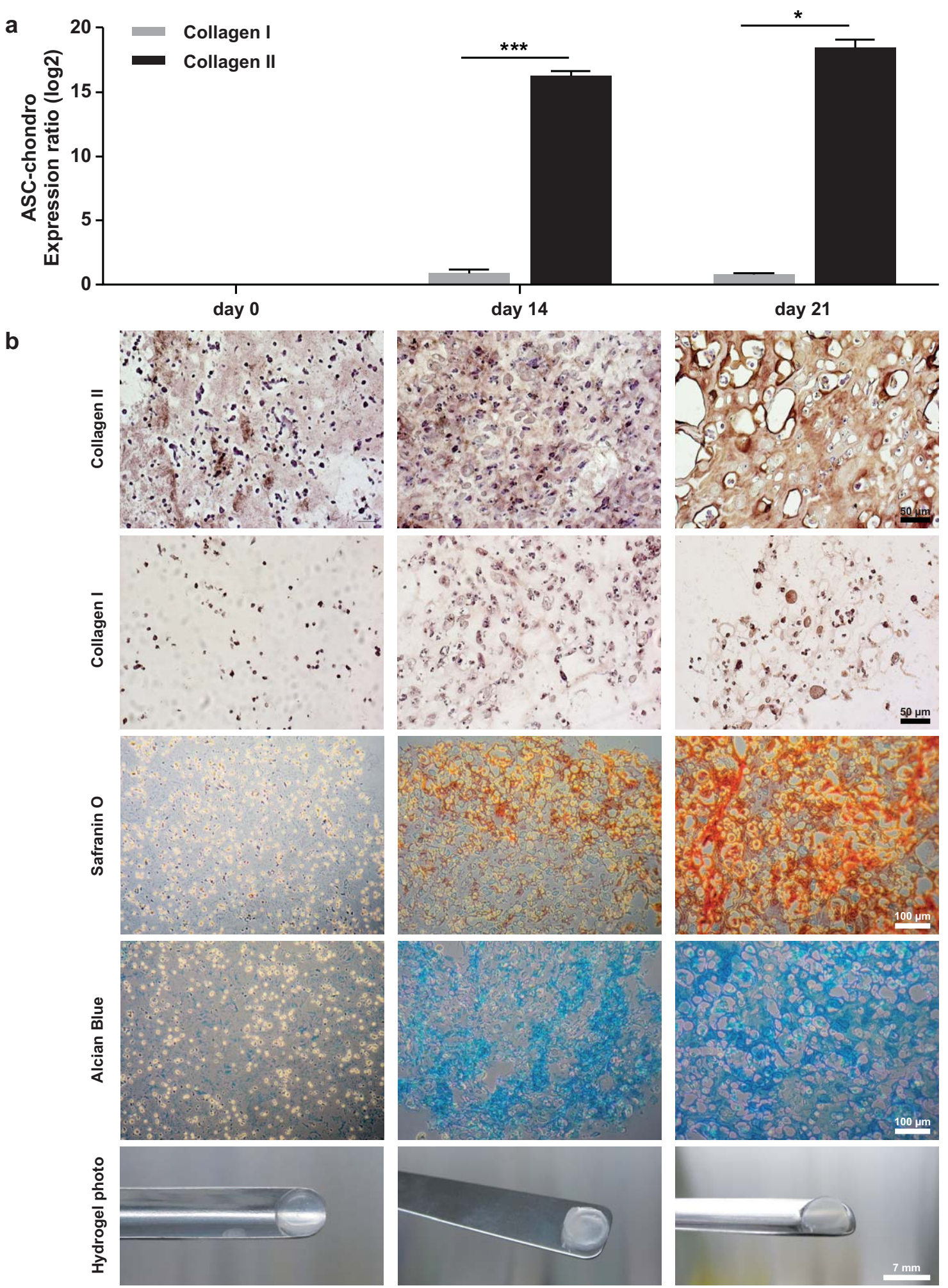

FIGURE 4. In vitro chondrogenesis of hASC encapsulated in GGMA $2 \% \mathrm{w} / \mathrm{N}$. (a) Gene expression ratio normalized to day 0 . ${ }^{*} p<0.05$, ${ }^{* * *} p<0.001$. (b) Histological analysis and macroscopic imaging of hydrogel along in vitro culture.

represents superior repair. Inter observer differences were assessed and no statistical differences were observed. Immunolocalization of rabbit collagen type II and collagen type I (Fig. 5) was performed to further characterize cartilage formed within the lesion site. Treatment of chondral lesions with GGMA + rASC (Fig. 5, top) allowed restoration of cartilage thickness, integration/bonding with native cartilage, as well as intense and reasonably homogenous 


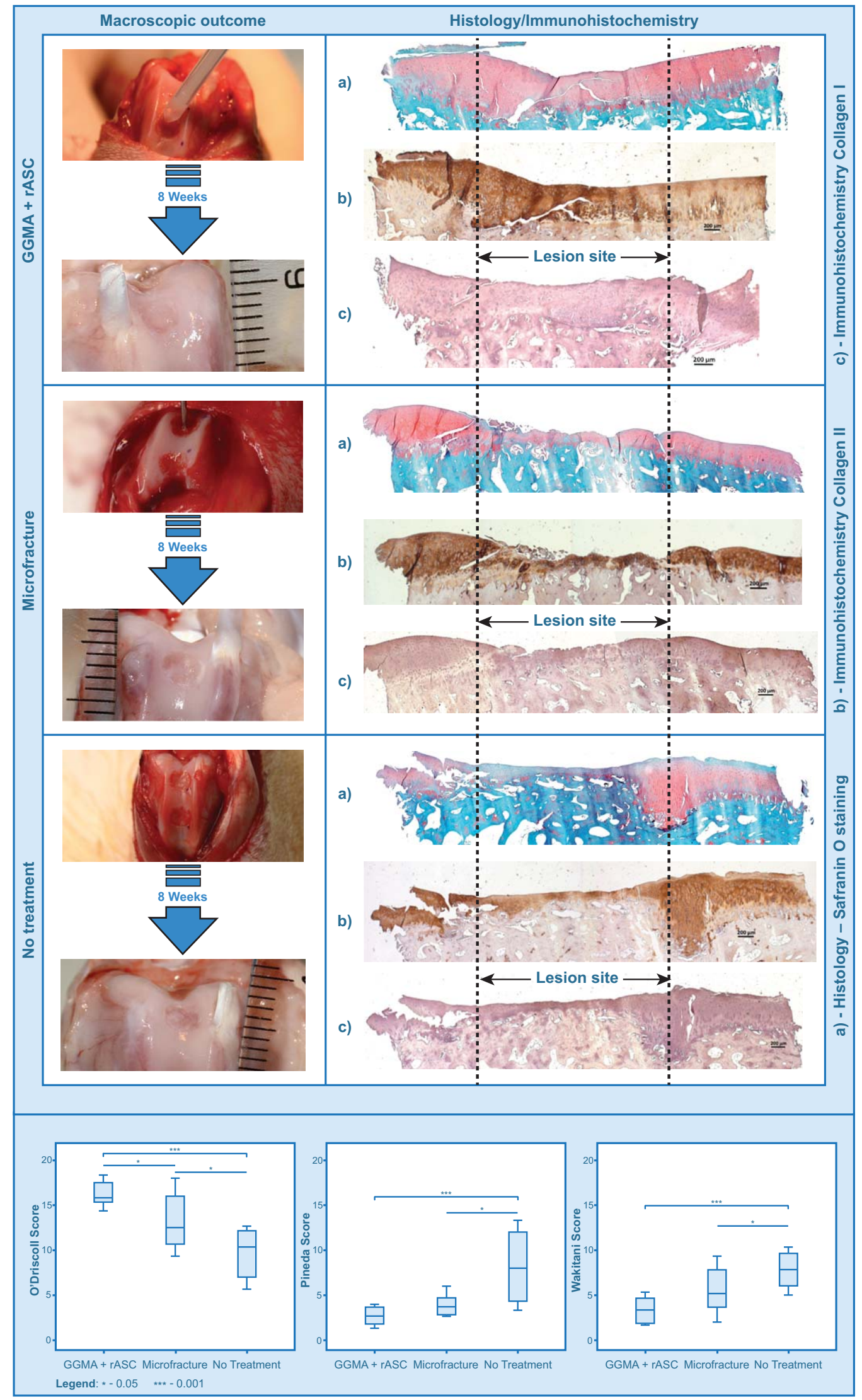

FIGURE 5. In vivo chondrogenesis of hASC encapsulated in GGMA $2 \%$ w/V:.Top panel. Histological analysis and macroscopic imaging of experimental groups after 8 weeks of implantation. Bottom panel. Histological scoring according to O'Driscoll, Pineda, and Wakitani scores. ${ }^{*} p<0.05$, $* * * p<0.001$. 
staining of ECM throughout the lesion site. Quantitative assessment of repair by all three scoring systems indicates significant improvement in cartilage repair as compared with the untreated lesions $(p<0.001)$. According to the O'Driscoll score, GGMA + rASC treatment also outperformed MFX $(p<0.05)$. Lesions treated with MFX (Fig. 5, Middle) demonstrated overgrowth of the subchondral bone into the lesion site which was covered by a thin layer of cartilaginous matrix stained by safranin $\mathrm{O}$ and collagen type II. This layer is irregular and bonding with adjacent native cartilage is incomplete. Nevertheless, the extent of cartilage repair by MFX was superior to untreated lesions, independently of the scoring system used $(p<0.05)$. The bottom image represents histological assessment of untreated lesions, whereas overgrowth of subchondral bone was evident and covered by a thin regular tissue. Herein, very limited cartilaginous matrix was formed, as indicated by the lack of safranin 0/ fast green and reduced collagen type II staining at the top layer of the tissue. Collagen type I deposition was negligible in all groups, yet expressed slightly higher in the untreated defects.

\section{DISCUSSION}

GG polysaccharide offers attractive features and characteristics for this particular application due to its aqueous solubility and viscous properties at physiological temperature, which makes it appealing for implementation of cell combination and surgical implantation protocols. In addition, the crosslinking by physiological ions leads to formation of a stable 3D structure and subsequent cell retention. In this regard, functionalization of the GG molecule by methacrylation extends solubility, allows control of spatiotemporal crosslinking, which combined extends flexibility of combination and implantation procedures and fine-tuning of hydrogel stiffness. For instance, at $2 \% \mathrm{w} / \mathrm{V}$, GGMA presents increased storage modulus as compared with the unmodified polysaccharide, $89.5 \pm 7.4$ and $56.2 \pm 1.4 \mathrm{kPa}$, respectively. ${ }^{22}$ Matrix stiffness, as a result of increased concentration or biochemical cues, has been reported to influence stem cell fate and particularly chondrogenesis. ${ }^{35}$ Within the context of this study, cells cultured within the GGMA 2\% w/V hydrogel shown improved cell metabolic activity, viability, and healthy expression of chondrogenic markers as compared with the least concentrated $(1 \% \mathrm{w} / \mathrm{V})$ or non-functionalized matrix.

$\mathrm{PoC}$ in the rabbit model allowed evaluation of the cartilage repair potential of the GGMA $2 \% \mathrm{w} / \mathrm{V}$ hydrogel as compared with MFX treatment. Currently considered as a goldstandard treatment, MFX still has limitations on what concerns the quality of the regenerated tissue, which can ultimately lead to treatment failure upon recurrence of symptoms. ${ }^{36,37}$ In this study, MFX group outcome showed formation of a thin layer of chondral tissue concurrent with subchondral bone overgrowth and an irregular surface. Such repair outcome is likely to disfavor adequate load bearing as well as smooth, pain-free joint motion. ${ }^{14,38,39} \mathrm{~A}$ different outcome was observed for lesions treated with
GGMA-rASC-cartilage thickness was maintained equivalently to adjacent native tissue while avoiding bone overgrowth. A smooth chondral surface was obtained following this treatment and ECM staining demonstrated reasonably uniform distribution of collagen type II and GAGs (Fig. 5). This repair outcome is believed to support long-term quality of the tissue as opposed to MFX treatment. At the 8week time-point, statistical differences were obtained between these groups according to O'Driscoll scoring. Nevertheless, it is important to mention that quantitative scores with a broad numerical range such as the O'Driscoll system may increase the likelihood of finding statistically significant differences. ${ }^{34}$ Still, the adoption of O'Driscoll score in the context of this study is pertinent as, contrary to alternative scores, it assesses integration of the repair tissue with its surroundings. ${ }^{34}$ In this study, no additional fixation technique was used to retain the hydrogel within the lesion site and precise volume filling was achieved, avoiding the need for on-site shaping of the scaffolding structure, which could be an advantage as compared with other cellbased and tissue engineered cartilage products currently in clinical development. ${ }^{12}$ In addition, viscous and sol-gel transition properties of the tested GGMA 2\% w/V hydrogel allowed controlled delivery of the matrix containing autologous $\mathrm{ASC}^{14}$ directly to lesion site, which favored delivery and retention of cells in situ. This fact is of significant importance as cell retention at lesion site is one of the main indicators of success for lesion repair. ${ }^{12,40}$ Adoption of the rabbit animal model for cartilage repair studies has significant advantages due to availability, ease of handling, low cost and abundance of comparative literature, ${ }^{14,41}$ but poses challenges related to reduced thickness $(0.4 \pm 0.1 \mathrm{~mm}$ in the trochlear groove) and surface area of articular cartilage. This model is adequate for $\mathrm{PoC}$ studies during early stage development of new technologies, particularly on what concerns evaluation of fixation of implantable devices. ${ }^{32,33}$ In this regard, the fixation merits of GGMA-rASC combination was demonstrated in a particularly challenging environment. Heterogeneous outcomes were naturally observed yet bona fide chondral repair was obtained with this treatment group, which is attributed to GGMA-rASC combination alone. The analysis of non-treated lesions demonstrated a limited self-healing of the induced defect that was statistically inferior than GGMA-rASC treatment. The self-repair ability of cartilage lesions, which is commonly reported in the rabbit model ${ }^{42,43}$ has been minimized by implementation of chondral defects with critical-size for which penetration and damage to the subchondral bone plate was avoided.

The GGMA formulation successfully supported in vitro chondrogenesis of both mature and progenitor cartilageforming cells. In a rabbit model, controlled delivery of cells into chondral lesions was achieved, while adequate spatiotemporal crosslinking supported volumetric filling of cartilage lesions and in situ retention of cells. Following 8 weeks of treatment, the combination of GGMA-rASC, supported full thickness regeneration of critical size lesions, good integration/bonding with native cartilage. Such combination 
therapy exhibited highly favorable physicochemical characteristics and good biological performance which may support less invasive and complex surgical procedures for cartilage repair.

\section{REFERENCES}

1. Vilela CA, Correia C, Oliveira JM, Sousa RA, Reis RL, EspregueiraMendes J. Clinical management of articular cartilage lesions. In: Oliveira JM, Reis RL, editors. Regenerative Strategies for the Treatment of Knee Joint Disabilities. Cham: Springer International Publishing; 2017. p. 2953.

2. Brittberg $M$, Lindahl $A$, Nilsson $A$, Ohlsson $C$, Isaksson $O$, Peterson L. Treatment of deep cartilage defects in the knee with autologous chondrocyte transplantation. N Engl J Med 1994;331: 889-895.

3. Lindahl A. From gristle to chondrocyte transplantation: Treatment of cartilage injuries. Philos Trans R Soc Lond B Biol Sci 2015;370: 20140369

4. Ogura T, Mosier BA, Bryant T, Minas T. A 20-year follow-up after first-generation autologous chondrocyte implantation. Am J Sports Med 2017;45:2751-2761.

5. Nawaz SZ, Bentley G, Briggs TWR, Carrington RWJ, Skinner JA, Gallagher KR, Dhinsa BS. Autologous chondrocyte implantation in the knee: Mid-term to long-term results. J Bone Joint Surg Am 2014;96:824-830.

6. Cvetanovich GL, Riboh JC, Tilton AK, Cole BJ. Autologous chondrocyte implantation improves knee-specific functional outcomes and health-related quality of life in adolescent patients. Am J Sports Med 2017;45:70-76.

7. McNickle AG, L'Heureux DR, Yanke AB, Cole BJ. Outcomes of autologous chondrocyte implantation in a diverse patient population. Am J Sports Med 2009;37:1344-1350.

8. Mistry H, Connock M, Pink J, Shyangdan D, Clar C, Royle P, Court R, Biant LC, Metcalfe A, Waugh N. Autologous chondrocyte implantation in the knee: Systematic review and economic evaluation. Health Technol Assess 2017;21:1-294.

9. Harris JD, Siston RA, Pan X, Flanigan DC. Autologous chondrocyte implantation: A systematic review. J Bone Joint Surg Am 2010;92:2220-2233.

10. Niemeyer P, Pestka JM, Kreuz PC, Erggelet C, Schmal H, Suedkamp NP, Steinwachs M. Characteristic complications after autologous chondrocyte implantation for cartilage defects of the knee joint. Am J Sports Med 2008;36:2091-2099.

11. Matsiko A, Levingstone TJ, O'Brien FJ. Advanced strategies for articular cartilage defect repair. Materials (Basel) 2013;6:637668.

12. Huang BJ, Hu JC, Athanasiou KA. Cell-based tissue engineering strategies used in the clinical repair of articular cartilage. Biomaterials 2016:98:1-22.

13. Goyal D, Goyal A, Keyhani S, Lee EH, Hui JHP. Evidence-based status of second- and third-generation autologous chondrocyte implantation over first generation: A systematic review of level I and II studies. Arthroscopy 2013;29:1872-1878.

14. Vilela CA, Correia C, Oliveira JM, Sousa RA, Espregueira-Mendes $J$, Reis RL. Cartilage repair using hydrogels: A critical review of in vivo experimental designs. Acs Biomater Sci Eng 2015;1:726739 .

15. Mollon B, Kandel R, Chahal J, Theodoropoulos J. The clinical status of cartilage tissue regeneration in humans. Osteoarthritis Cartilage 2013;21:1824-1833.

16. Jeuken RM, Roth AK, Peters RJRW, van Donkelaar CC, Thies JC, van Rhijn LW, Emans PJ. Polymers in cartilage defect repair of the knee: Current status and future prospects. Polymers 2016;8: 219 .

17. Correia C, Pereira AL, Duarte ARC, Frias AM, Pedro AJ, Oliveira JT, Sousa RA, Reis RL. Dynamic culturing of cartilage tissue: The significance of hydrostatic pressure. Tissue. Eng Part A 2012;18: 1979-1991.

18. Oliveira JT, Gardel LS, Rada T, Martins L, Gomes ME, Reis RL. Injectable gellan gum hydrogels with autologous cells for the treatment of rabbit articular cartilage defects. J Orthop Res 2010; 28:1193-1199.
19. Oliveira JT, Martins L, Picciochi R, Malafaya PB, Sousa RA, Neves NM, Mano JF, Reis RL. Gellan gum: A new biomaterial for cartilage tissue engineering applications. J Biomed Mater Res A 2010; 93:852-863.

20. Silva-Correia J, Gloria A, Oliveira MB, Mano JF, Oliveira JM, Ambrosio L, Reis RL. Rheological and mechanical properties of acellular and cell-laden methacrylated gellan gum hydrogels. J Biomed Mater Res A 2013;101:3438-3446.

21. Silva-Correia J, Oliveira JM, Oliveira JT, Sousa RA, Reis RL. Photo-crosslinked Gellan gum-based hydrogels: Methods and uses thereof. US 2014/350237 (A1) - 2014-11-27.

22. Silva-Correia J, Oliveira JM, Caridade SG, Oliveira JT, Sousa RA, Mano JF, Reis RL. Gellan gum-based hydrogels for intervertebral disc tissue-engineering applications. J Tissue Eng Regen Med 2011;5:e97-107.

23. Rackwitz L, Djouad F, Janjanin S, Nöth U, Tuan RS. Functional cartilage repair capacity of de-differentiated, chondrocyte- and mesenchymal stem cell-laden hydrogels in vitro. Osteoarthritis Cartilage 2014;22:1148-1157.

24. Bernhard JC, Vunjak-Novakovic G. Should we use cells, biomaterials, or tissue engineering for cartilage regeneration? Stem Cell Res Ther 2016;7:56.

25. Garcia J, Mennan C, McCarthy HS, Roberts S, Richardson JB, Wright KT. Chondrogenic potency analyses of donor-matched chondrocytes and mesenchymal stem cells derived from bone marrow, infrapatellar fat pad, and subcutaneous fat. Stem Cells Int 2016;2016:6969726.

26. Stenberg J, de Windt TS, Synnergren J, Hynsjö L, van der Lee $J$, Saris DBF, Brittberg M, Peterson L, Lindahl A. Clinical outcome 3 years after autologous chondrocyte implantation does not correlate with the expression of a predefined gene marker set in chondrocytes prior to implantation but is associated with critical signaling pathways. Orthop J Sports Med 2014;2: 232596711455078

27. Kasir R, Vernekar VN, Laurencin CT. Regenerative engineering of cartilage using adipose-derived stem cells. Regen Eng Transl Med 2015;1:42-49.

28. Zuk PA, Zhu M, Mizuno H, Huang J, Futrell JW, Katz AJ, Benhaim P, Lorenz HP, Hedrick MH. Multilineage cells from human adipose tissue: Implications for cell-based therapies. Tissue Eng 2001;7:211-228.

29. Centeno CJ. Clinical challenges and opportunities of mesenchymal stem cells in musculoskeletal medicine. PM R 2014;6:70-77.

30. Zanata F, Shaik S, Devireddy RV, Wu X, Ferreira LM, Gimble JM. Cryopreserved adipose tissue-derived stromal/stem cells: Potential for applications in clinic and therapy. Adv Exp Med Biol 2016; 951:137-146.

31. Doner LW. Rapid purification of commercial gellan gum to highly soluble and gellable monovalent cation salts. Carbohydr Polym 1997;32:245-247.

32. Hurtig MB, Buschmann MD, Fortier LA, Hoemann CD, Hunziker $E B$, Jurvelin JS, Mainil-Varlet $P$, Mcllwraith CW, Sah $R L$, Whiteside RA. Preclinical studies for cartilage repair: Recommendations from the international cartilage repair society. Cartilage 2011;2:137-152.

33. International A. ASTM F2451-05(2010), Standard guide for in vivo assessment of implantable devices intended to repair or regenerate articular cartilage, ASTM International, West Conshohocken, PA, 2010, www.astm.org.

34. Rutgers M, van Pelt MJP, Dhert WJA, Creemers LB, Saris DBF. Evaluation of histological scoring systems for tissue-engineered, repaired and osteoarthritic cartilage. Osteoarthritis Cartilage 2010; 18:12-23.

35. Wang T, Lai JH, Han L-H, Tong $X$, Yang F. Chondrogenic differentiation of adipose-derived stromal cells in combinatorial hydrogels containing cartilage matrix proteins with decoupled mechanical stiffness. Tissue Eng A 2014;20:2131-2139.

36. Erggelet $C$, Vavken P. Microfracture for the treatment of cartilage defects in the knee joint - A golden standard? J Clin Orthop Trauma 2016;7:145-152.

37. Gracitelli GC, Moraes VY, Franciozi CE, Luzo MV, Belloti JC. Surgical interventions (microfracture, drilling, mosaicplasty, and allograft transplantation) for treating isolated cartilage defects of the knee in adults. Cochrane Database Syst Rev 2016;9:CD010675. 
38. Ulstein $\mathrm{S}$, Årøen $\mathrm{A}$, Røtterud JH, Løken $\mathrm{S}$, Engebretsen L, Heir S. Microfracture technique versus osteochondral autologous transplantation mosaicplasty in patients with articular chondral lesions of the knee: A prospective randomized trial with longterm follow-up. Knee Surg Sports Traumatol Arthrosc 2014;22: 1207-1215.

39. Marcacci M, Filardo G, Kon E. Treatment of cartilage lesions: What works and why? Injury 2013;44:S11-S15.

40. Man Z, Hu X, Liu Z, Huang H, Meng Q, Zhang X, Dai L, Zhang J, Fu $X$, Duan X, Zhou C, Ao Y. Transplantation of allogenic chondrocytes with chitosan hydrogel-demineralized bone matrix hybrid scaffold to repair rabbit cartilage injury. Biomaterials 2016;108:157-167.

41. Chu CR, Szczodry M, Bruno S. Animal models for cartilage regeneration and repair. Tissue Eng B Rev 2010;16:105-115.

42. Shapiro F, Koide S, Glimcher MJ. Cell origin and differentiation in the repair of full-thickness defects of articular cartilage. J Bone Joint Surg Am 1993;75:532-553.

43. Ahern BJ, Parvizi J, Boston R, Schaer TP. Preclinical animal models in single site cartilage defect testing: A systematic review. Osteoarthritis Cartilage 2009;17:705-713. 